\section{Der Einfluss von Infektionen auf die Entstehung von Asthma bronchiale im Kindesalter}

Die Entstehung von Asthma bronchiale im Kindesalter wird schon seit langem mit dem Auftreten von Atemwegsinfektionen in Verbindung gebracht. Im Folgenden soll die Rolle von Infektionen bei der Auslösung akuter obstruktiver Atemwegssymptome und ihr Einfluss auf die Entstehung allergischen Asthmas behandelt werden.

\section{Akute bronchiale Obstruktion und Asthmaexazerbation durch virale Atemwegsinfektionen}

Virusinfektionen der Atemwege sind im Kindesalter ein wichtiger Auslöser obstruktiver Atemwegserkrankungen $[1,2]$. Etwa $80 \%$ der Asthmaepisoden im Schulalter sind mit Virusinfektionen der oberen Atemwege assoziiert [3], wobei Rhinoviren (RV) die häufigsten Erreger $(>60 \%)$ in dieser Altersgruppe sind [4]. Bei Kindern unter 2 Jahren ist vor allem Respiratory Syncytial Virus (RSV) (50 - 60\%) mit obstruktiven Atemwegserkrankungen assoziiert [5,6]. Auch Enteroviren, Koronaviren, Parainfluenza-Viren (PIV) und Adenoviren werden bei Asthmaexazerbationen im Kindesalter nachgewiesen [5,7]. Im Gegensatz zu den Atemwegsviren scheinen bakterielle Infektionen der Atemwege auch mit atypischen Bakterien wir Chlamydia pneumoniae nicht mit Asthmaexazerbationen assoziiert zu sein [5]. Ein erhöhtes Risiko obstruktive Atemwegssymptome bei viralen Atemwegsinfektionen zu entwickeln wurde sowohl für Kinder mit verminderter Lungenfunktion und für tabakrauchexponierte Kinder gezeigt als auch für Kinder mit Atopie [8-12].

Auf welchem Wege Virusinfektionen der Atemwege eine Atemwegsobstruktion auslösen wird nur unvollständig verstanden. Durch humane Studien und in Tiermodellen haben sich jedoch Hinweise auf mögliche Pathomechanismen ergeben. Es ist unumstritten, dass RSV, Influenza-Virus und PIV Infektionen der unteren Atemwege verursachen können. Kürzlich konnte aber durch mRNA-Nachweis auch gezeigt werden, dass RV, ein typischer Erkältungserreger der oberen Atemwege, auch die unteren Atemwege infizieren kann [13]. Eine solche Infektion, die primär das respiratorische Epithel betrifft, führt zu einer Entzündungsreaktion der betroffenen Atemwege. Nach experimenteller RV-Infektion zeigte sich in Bronchialschleimhautbiopsien eine submuköse Infiltration mit Lymphozyten und eine Zunahme intraepithelialer eosinophiler Granulozyten [14]. Bei der schweren RSV-Bronchiolitis

Pneumologie 2000; 54: 401-406

(C) Georg Thieme Verlag Stuttgart · New York ISSN 0934-8387
J. Schwarze

Klinik für Kinder- und Jugendmedizin im St. Josef-Hospital

Bochum, Universitätsklinik Ruhr-Universität Bochum zeigen pathologische Präparate eine starke entzündliche Reaktion vor allem der Bronchiolen mit Desquamation und Pfröpfen aus Fibrin und Zelldetritus [15]. Es kommt zu Atelektasen und Überblähungsbezirken [16]. Die Entzündungsreaktion wird von neutrophilen Granulozyten und mononukleären Zellen dominiert [17]. Es werden aber auch eosinophile Granulozyten beobachtet [16]. Auch bei leichteren RSV-Infektionen gibt es Hinweise auf eine Rekrutierung und Aktivierung eosinophiler Granulozyten in den Atemwegen: bei Kindern mit obstruktiver RSV-Bronchitis/ Bronchiolitis wurden im Nasopharyngealsekret erhöhte ECP-Spiegel beobachtet, die mit dem Schweregrad der Erkrankung korrelierten [18]. Parallel dazu kam es im peripheren Blut zu einem Anstieg der Eosinophilenzahl [19]. Auch in vitro lässt sich eine Aktivierung eosinophiler Granulozyten durch RSV demonstrieren [20]. Diese Entzündung ist Teil und Konsequenz einer immunologischen Reaktion auf die Virusinfektion der Atemwege. Angestoßen wird sie unter anderem durch die Sekretion proinflammatorischer Zytokine wie IL-6, IL-8, IL-11, GMCSF und Rantes durch das infizierte Epithel [21-23]. Es gibt zahlreiche Hinweise darauf, dass T-Lymphozyten an der Steuerung dieser Immunantwort entscheidend beteiligt sind. Die experimentelle RV-Infektion ist mit einer Lymphopenie im peripheren Blut assoziiert [24], die mit dem Schweregrad der Erkältung und der Atemwegshyperreaktivität (AHR) korreliert [14]. Zudem kommt es zu einem Anstieg der Lymphozytenzahl im Nasensekret und im Epithel der unteren Atemwege $[14,25]$ und es lässt sich sowohl eine antigenspezifische als auch eine antigenunabhängige Aktivierung von T-Zellen feststellen [26].

Für das Verständnis der Pathomechanismen bei der virusinduzierten Immunantwort sind Tiermodelle von großer Bedeutung. In einem Mausmodell führt die akute RSV-Infektion zu AHR nach Methacholinprovokation und zu einer Atemwegsentzündung mit neutrophilen und eosinophilen Granulozyten. Dies ist mit einer gesteigerten Produktion von IFN- $\gamma$, bei erhaltener IL-5-Produktion, assoziiert [27]. In diesem Modell konnte durch Depletion während der Infektion gezeigt werden, dass die AHR, die pulmonale Eosinophilie und ein Anstieg von IL-5 in der bronchoalveolären Lavage von der Anwesenheit von CD8+-T-Lymphozyten abhängig sind [28]. Virusinfektionen rufen in erster Linie zytotoxische IFN- $\gamma$ produzierende $\mathrm{CD}^{+}-\mathrm{T}-Z$ ellen hervor, aber es treten auch virusspezifische nichtzytotoxische IL-5 produzierende CD8+T-Zellen auf [29]. Letztere scheinen eine zentrale Rolle bei Entstehung der virusinduzierten eosinophilen Komponente der Atemwegsentzündung und der AHR zu spielen. Durch Verwendung von „knock-out“-Mäusen für die Zytokine IL-5, 
IL-4 und IFN- $\gamma$ wurde zudem gezeigt, dass IL-5, nicht aber IL4 oder IFN- $\gamma$, notwendig sind für die Entstehung der RSVinduzierten AHR und der pulmonalen Eosinophilie [30]. Die neutrophile Komponente der Atemwegsentzündung blieb durch das Fehlen von IL-5 unbeeinflusst. Blockade der Migration eosinophiler Granulozyten in die Atemwege mittels eines anti-VLA4-Antikörpers verhindert die Entstehung der AHR. Ein direkter Zusammenhang zwischen eosinophiler Entzündung und AHR ist somit sehr wahrscheinlich. Diese Studien zeigen die zentrale Bedeutung von T-Zellen bei der Steuerung, von IL-5 als Mediator und von eosinophilen Granulozyten als Effektoren bei der virusinduzierten AHR. Bei Meerschwein und Ratte können die von eosinophilen Granulozyten freigesetzten kationischen Proteine präsynaptische M2-Muskarinrezeptoren parasympathischer Nerven blockieren und somit einen inhibitorischen Feed-back-Mechanismus für die Acetylcholinfreisetzung unterbrechen [31,32]. Virale Neuraminidasen können auch ohne eosinophile Entzündung direkt M2Muskarinrezeptoren blockieren [33]. Es kommt darauf hin zu Bronchokonstriktion durch eine erhöhte Acetylcholinausschüttung. Neben dem inflammatorischen Weg sind auch andere Pathomechanismen der virusinduzierten Asthmaentstehung denkbar. Durch eine Virusinfektion können Funktionen des respiratorischen Epithels gestört werden. Im Meerschwein wurde bei viraler Atemwegsinfektion eine verminderte NO-Produktion durch das Epithel beobachtet, die mit AHR assoziiert war [34]. NO ist der relaxierende Faktor des nicht-adrenergen-nicht-cholinergen inhibitorischen Systems der Bronchialmuskulatur, welches auch bei der RSV-Infektion der „Cotton Rat“ gestört ist [35]. Die Aktivität der neutralen Endopeptidase des respiratorischen Epithels ist bei Virusinfektionen vermindert, wie bei Ratte und Meerschwein gezeigt wurde [36 - 39]. Dieses Enzym baut Neuropeptide wie Substanz P und Neurokinin A ab, welche die Leukotriensynthese stimulieren [40], Mastzellmediatoren freisetzen [41], die Mukusproduktion erhöhen [42] und eine Kontraktion glatter Muskulatur hervorrufen [43]. Eine verminderte Barrierenfunktion des infizierten Epithels kann zur Stimulation sensorischer C-Fasern der Bronchialwand führen, die über einen Hirnstammreflex und durch Freisetzen von Substanz P und Neurokinin A Bronchokonstriktion hervorrufen können $[26,43]$. Die Rolle der sensorischen C-Fasern für den Menschen ist jedoch nicht klar: Im humanen Modell der RVInfektion zeigte sich keine erhöhte AHR nach Provokation mit Bradykinin, das über eine Stimulation der sensorischen CFasern wirkt [44]. Das Zusammenspiel von peribronchialer Entzündung, Ödem und einer vermehrten Transsudation beeinträchtigt vor allem die Funktion der kleinlumigen peripheren Atemwege, wo es durch eine Atemwegs-ParenchymEntkoppelung noch zusätzlich zur Lumeneinengung kommen kann [45]. Diese führt zu Überblähung und einer Störung des Gasaustausches. Die hier diskutierten Pathomechanismen virusinduzierter obstruktiver Atemwegssymptome sind in Tab. 1 zusammengestellt.

\section{Infektionen als Schutz vor allergischer Sensibilisierung und Asthmaentstehung}

Neben der akuten Auslösung obstruktiver Atemwegssymptome durch virale Atemwegsinfektionen gibt es auch Hinweise darauf, dass Infektionen Einfluss haben auf die Wahrscheinlichkeit, zu einem späteren Zeitpunkt im Kindesalter allergisches Asthma zu entwickeln. Es gibt eine Reihe von
Tab. 1 Pathogenetische Faktoren virusinduzierter obstruktiver Atemwegssymptome

\begin{tabular}{|c|c|}
\hline Pathomechanismus & wesentliche Faktoren \\
\hline $\begin{array}{l}\text { Immunantwort auf } \\
\text { RSV-Infektion }\end{array}$ & $\begin{array}{l}\text { CD8+-T-Lymphozyten } \\
\text { Interleukin-5 }\end{array}$ \\
\hline Atemwegsentzündung & $\begin{array}{l}\text { eosinophile Granulozyten } \\
\text { neutrophile Granulozyten } \\
\text { Lymphozyten }\end{array}$ \\
\hline $\begin{array}{l}\text { gesteigerte Acetylcholin- } \\
\text { Freisetzung }\end{array}$ & $\begin{array}{l}\text { Dysfunktion der M2-Muskarinrezep- } \\
\text { toren durch } \\
\text { direkte Viruseinwirkung } \\
\text { Major Basic Protein aus Eosinophilen }\end{array}$ \\
\hline verminderte NO-Produktion & $\begin{array}{l}\text { Epitheldysfunktion } \\
\text { führt zu verminderter Relaxation der } \\
\text { Atemwegsmuskulatur }\end{array}$ \\
\hline $\begin{array}{l}\text { gesteigerte Neuropeptid- } \\
\text { Wirkung }\end{array}$ & $\begin{array}{l}\text { vermehrte Ausschüttung von Substan- } \\
\text { ce } \mathrm{P} \text { und Neurokinin } \mathrm{A} \text { aus C-Fasern } \\
\text { verminderte Aktivität der neutralen } \\
\text { Endopeptidase }\end{array}$ \\
\hline $\begin{array}{l}\text { Lumeneinengung der } \\
\text { peripheren Atemwege }\end{array}$ & $\begin{array}{l}\text { entzündliche Infiltrate } \\
\text { Ödem } \\
\text { vermehrte Exsudation } \\
\text { Atemwegs-Parenchym-Entkoppelung }\end{array}$ \\
\hline
\end{tabular}

epidemiologischen Beobachtungen, die darauf hinweisen, dass Infektionen das Risiko der allergischen Sensibilisierung der Atemwege vermindern können. So wurde nach einer Masernepidemie in Guinea-Bissau eine verminderte AtopiePrävalenz beobachtet [46]. In einer japanischen Untersuchung zeigte sich eine geringere Rate von Asthma und Allergien bei BCG-geimpften Kindern mit stark positivem Tuberkulintest [47], der möglicherweise durch Tuberkulose-Exposition bedingt war. Darüber hinaus bestand bei Personen mit einer starken Tuberkulinreaktion oder einer Tuberkulinkonversion eine größere Wahrscheinlichkeit, dass sie ihr Asthma verlieren, als bei Personen mit einer negativen Tuberkulinreaktion. Auch der „Geschwistereffekt“ und der „Bauerneffekt“ auf die Atopieentwicklung werden im Zusammenhang mit der Infektionsexposition gesehen. Zahlreiche Studien haben gezeigt, dass das Risiko an allergischen Symptomen zu leiden mit steigender Zahl älterer Geschwister sinkt $[48,49]$, und dass dieses Risiko bei Bauernkindern geringer ist als bei Kindern aus Nichtbauernfamilien des gleichen Ortes [50]. Bei Bauernkindern korreliert das Risiko der allergischen Sensibilisierung negativ mit der Häufigkeit des Stalltierkontaktes. Beide Beobachtungen werden damit erklärt, dass häufige Infektionen vermittelt durch ältere Geschwister oder durch Kontakt zu Stalltieren immunologische Reaktionen im Sinne einer TH-1-Antwort auslösen, durch die TH-2-Immunantworten wie bei der allergischen Sensibilisierung unterdrückt werden.

Auch in Tiermodellen wurde die Hypothese überprüft, dass die Abnahme von Infektionen als Teil des sog. „western life style“ zu dem Anstieg von Asthma und Allergien beiträgt, der in den industrialisierten Ländern beobachtet wird. Insbesondere mykobakteriellen Infektionen wird angesichts der oben genannten epidemiologischen Untersuchungen eine diesbezügliche Rolle zugeschrieben, zumal die Immunantwort bei der Tuberkulose den Prototyp der TH-1-Reaktion darstellt, die 
durch die Produktion der Zytokine IFN- $\gamma$ und IL-12 gekennzeichnet ist, und die als verzögerte zelluläre Immunreaktion (Typ IV nach Gell und Coombs) mit einer durch zytotoxische NK-Zellen, Epitheloidzellen und Makrophagen vermittelten Effektorphase verläuft. Auch im Mausmodell erzeugen Mykobakterien, wie zum Beispiel BCG, eine anhaltende IFN- $\gamma$ Produktion. Eine Behandlung von BALB/c-Mäusen mit BCGBakterien vor einer allergischen Sensibilisierung mit Ovalbumin vermindert die Bildung allergenspezifischer IgE und IgG1 Antikörper im Serum der Mäuse, reduziert die Konzentrationen der TH-2 Zytokine IL-4 und IL-5 in der bronchoalveolären Lavage und verhindert die Entstehung der allergischen Atemwegsentzündung sowie der Atemwegshyperreaktivität. Diese Beobachtungen waren in mehreren Untersuchungen verschiedener Autoren abhängig von der Dosis, der zeitlichen Aufeinanderfolge und dem Applikationsweg der BCG-Bakterien. Virulente Keime waren nicht in jedem Fall dafür erforderlich, sondern der Effekt ließ sich auch bei abgetöteten Bakterien nachweisen [51-53]. Diese Untersuchungen im Tiermodell stärken die Hypothese, dass ein Zusammenhang zwischen mykobakteriellen Infektionen und der Unterdrückung einer allergischen Reaktion besteht.

\section{Infektionen mit Atemwegsviren und atypischen Bakterien als Risikofaktor für die Entstehung des allergischen Asthmas}

Andere Infektionen begünstigen aber möglicherweise die Entstehung von Asthma. Bei Kindern und Erwachsenen mit Asthma wurden häufiger chronische Infektionen mit Mykoplasma pneumoniae [54] und Chlamydia pneumoniae [5] festgestellt als bei Gesunden. Eine Infektion mit diesen atypischen Bakterien korreliert mit der Schwere der Atemwegsentzündung und bei besiedelten Patienten führt eine Therapie mit Clarithromycin, einem Makrolid, zu einem Rückgang proinflammatorischer Zytokine [55]. Mehrere epidemiologische Beobachtungen legen nahe, dass Virusinfektionen der Atemwege neben ihren akuten Effekten auch das Risiko der Entstehung allergischen Asthmas anheben können. Bei Kindern aus Atopikerfamilien wurde eine erhöhte Rate von allergischer Sensibilisierung gegen Aeroallergene 1-2 Monate nach viralen Atemwegsinfektionen beobachtet [56]. In einer prospektiven Kohortenstudie waren zur Hospitalisierung führende RSV-Infektionen im Säuglingsalter der wichtigste Risikofaktor für das Auftreten von Asthma bronchiale und für die Entstehung einer allergischen Sensibilisierung in den ersten 3 Lebensjahren [57]. Eine atopische Familienanamnese erhöhte dieses Risiko weiter. Andere kontrollierte Längsschnittstudien bei Kindern, die im Säuglingsalter eine RSVBronchiolitis hatten, zeigen eine vorübergehende Zunahme von obstruktiven Atemwegssymptomen (wheezing) und von AHR, die jedoch nicht mit einer erhöhten Rate allergischer Sensibilisierung verbunden waren [58-60]. Alle diese Studien haben das Problem eines Selektions-Bias bei der Wahl der Stichprobe, denn sie untersuchen Patienten, die wegen einer RSV-Bronchiolitis hopitalisiert wurden, was nur bei einer kleinen Minderheit von RSV-infizierten Kindern notwendig ist bei einer Durchseuchungsrate mit RSV von fast 100\%. Ergebnisse aus der Tucson Children's Respiratory Study, einer prospektiven Studie einer Geburtskohorte ohne SelektionsBias, zeigen nach RSV-Infektion in den ersten 3 Lebensjahren ein erhöhtes Risiko für obstruktive Atemwegssymptome im Alter von 6 Jahren, welches mit 13 Jahren nicht mehr erhöht ist [61]. Auch hier bestand keine Assoziation mit der Häufigkeit von allergischer Sensibilisierung. Die Frage, ob virale Atemwegsinfektionen selbst tatsächlich einen prädisponierenden Faktor für eine allergische Sensibilisierung über die Atemwege darstellen oder ob sie eine genetische Prädisposition zur Atopie früh demaskieren, ist nicht geklärt. Weitere Ergebnisse der Tucson Children's Respiratory Study zeigen, dass die Mehrzahl der Kinder mit obstruktiven Atemwegssymptomen im Kleinkindesalter mit 6 Jahren kein Asthma mehr haben [11]. Diese Gruppe hat schon bei Geburt eine reduzierte Lungenfunktion $[9,10]$. Bei einer nicht geringen Zahl von Kindern persistiert das Asthma jedoch bis zum 11 Lebensjahr [62]. In dieser Gruppe sind Atopiemarker erhöht und es lässt sich eine spezifische allergische Sensibilisierung nachweisen [12]. Zusammen betrachtet sprechen diese Ergebnisse eher dafür, dass virale Atemwegsinfektionen eine frühzeitige Asthmaentstehung bei atopischer Prädisposition auslösen können, jedoch selbst nicht die Entstehung eines allergischen Asthmas induzieren. In jedem Fall ist es jedoch wichtig, die Pathomechanismen aufzuklären, durch die die Asthmaentwicklung angestoßen oder begünstigt wird, um präventive therapeutische Strategien für Kinder aus Risikogruppen entwickeln zu können.

Beim Meerschweinchen und bei der Maus lässt sich nach PIVoder RSV-Infektion eine verstärkte allergische Sensibilisierung über die Atemwege mit eosinophiler Entzündung und AHR hervorrufen [63-65]. In diesen Modellen wurde jeweils eine erste Allergenexposition während der akuten Infektion durchgeführt, was zu einer erhöhten Allergenresorption über das durch Infektion geschädigte Epithel und damit zu einer verstärkten Sensibilisierung mit höheren spezifischen $\operatorname{IgE}$ Spiegeln führte als bei nicht infizierten Tieren. Im Mausmodell treten AHR und eine Atemwegsentzündung mit eosinophiler Komponente aber auch auf, wenn eine schwache allergische Sensibilisierung über die Atemwege erst nach Abklingen einer vorhergehenden RSV-Infektion erfolgt [27]. In diesem Fall kann nicht von einer erhöhten Allergenresorption ausgegangen werden, zumal die allergenspezifischen $\operatorname{IgE}$ Spiegel sich zwischen infizierten und nicht infizierten Tieren nicht unterscheiden. Es ist vielmehr wahrscheinlich, dass durch die RSV-Infektion eine Immunantwort ausgelöst wird, die auch zu einem späteren Zeitpunkt eine allergische Sensibilisierung über die Atemwege begünstigt. Diese These wird durch folgende Beobachtungen gestützt. Die Abwesenheit von IL-5 während der akuten RSV Infektion führt - vermutlich durch das Fehlen einer eosinophilen Entzündungsreaktion zu einer Verminderung der AHR und der Lungeneosinophilie nach der in Gegenwart von IL-5 erfolgten allergischen Sensibilisierung $[30,66]$. Eine Entzündung mit eosinophiler Komponente während der RSV-Infektion scheint also wegbereitend zu sein für die verstärkte Entzündung und AHR bei nachfolgender Sensibilisierung. Dazu kommt, dass sich der Effekt der RSV-Infektion auf nicht RSV-infizierte Tiere übertragen lässt durch adoptiven Transfer von CD8+-T-Lymphozyten, nicht aber durch CD4+-T-Zellen [67]. Es kommt dabei nach der auf den Transfer folgenden Sensibilisierung zu AHR und zur Lungeneosinophilie wie bei RSV-infizierten Tieren und zu einem Anstieg der IL-5-Produktion. Interessanterweise ist das Ergebnis des Transfers von dem Zeitpunkt nach RSVInfektion abhängig, an dem er durchgeführt wird. Ein Transfer von T-Zellen 14 Tage nach RSV-Infektion führt $\mathrm{zu}$ den beschriebenen Konsequenzen, wohingegen es bei einem $\mathrm{T}$ - 
Zell-Transfer 7 Tage nach Infektion weder zu AHR, noch zu Lungeneosinophilie oder einem Anstieg der IL-5-Produktion nach allergischer Sensibilisierung kommt. Dies legt nahe, dass während der akuten Infektion (Tag 7) eine TH-1-Immunantwort überwiegt und IFN- $\gamma$-produzierende, zytotoxische CD8 ${ }^{+}-$ T-Zellen übertragen werden, während nach Abklingen der Infektion (Tag 14) eine TH-2-Immunreaktion in den Vordergrund rückt und der Transfer von nicht zytotoxischen, IL-5 produzierenden $\mathrm{CD}^{+}-\mathrm{T}-Z$ Zellen eine allergische Sensibilisierung mit eosinophiler Entzündung begünstigt. Dass virale Atemwegsinfektionen eine mehrschichtige Immunantwort einleiten, ist gut denkbar, da diese durch unterschiedliche Antigene des gleichen Erregers ausgelöst wird. Es wurden RSV-Antigene identifiziert, die bei RSV-Infektion nach Vakzination unterschiedliche Immunantworten auslösen. So ruft das G-Protein eine starke TH-2-Antwort mit eosinophiler Entzündung und schwerer Erkrankung hervor, während das F-Protein zu einer milderen TH-1-Reaktion führt [68]. Zusammenfassend ist festzustellen, dass die RSV-Infektion im Tiermodell eine allergische Sensibilisierung über die Atemwege begünstigen kann. Dies geschieht zum einen durch eine gesteigerte Allergenaufnahme, zum anderen aber auch durch die Induktion einer Immunantwort mit vermehrter IL-5Bildung, welche eine allergische Entzündung und die daraus resultierende AHR fördert. Abb.1 stellt eine Hypothese der immunologischen Zusammenhänge zwischen RSV-Infektion, allergischer Sensibilisierung über die Atemwege und AHR dar, die sich aus den oben genannten Beobachtungen ergibt.

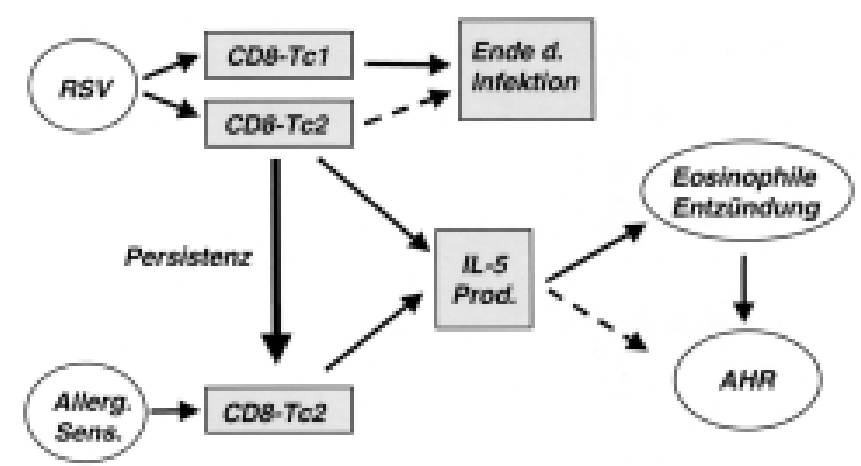

Abb. 1 Bei der RSV-Infektion treten zum einen zytotoxische IFN- $\gamma$ produzierende CD8+-T-Lymphozyten (CD8-Tc1) auf, die für die Bekämpfung der Infektion von zentraler Bedeutung sind, und es werden zum anderen IL-4- und IL-5-produzierende CD8+-T-Lymphozyten (CD8-Tc2) aktiviert. Letztere können die Infektion überdauern und werden bei einer allergischen Atemwegssensibilisierung reaktiviert. Sie können sowohl bei der akuten RSV-Infektion als auch bei einer allergischen Sensibilisierung nach einer RSV-Infektion eine gesteigerte Produktion von IL-5 induzieren, was die Entstehung einer eosinophilen Entzündung der Atemwege sowie das Auftreten von AHR begünstigt.

\section{Ausblick}

Durch die oben aufgeführten immunologischen Beobachtungen werden präventive Strategien zur Vermeidung von Asthmaentwicklung nach viralen Atemwegsinfektionen denkbar. Mögliche Konzepte sind eine anti-IL-5-Antikörper- Behandlung während schwerer viraler Atemwegsinfekte mit obstruktiven Symptomen wie der RSV-Bronchiolitis, eine „TH-1Therapie“ im Anschluss an solche Infektionen z.B. mit topi- schem IL-12 [69] oder durch Immunstimulation mit CpGOligonukleotiden [70], die der mykobakteriellen DNA entstammen, sowie eine Vakzination gegen „Asthma induzierende“ virale Antigene, durch die die Immunantwort auf diese Antigene moduliert wird. Bevor neue therapeutische Konzepte zur Anwendung kommen können ist es notwendig, dass Risikogruppen für eine virusinduzierte Asthmaentwicklung durch weitere epidemiologische Untersuchungen genauer charakterisiert werden.

\section{Literatur}

${ }^{1}$ Busse WW. Respiratory infections: Their role in airway responsiveness and the pathogenesis of asthma. J Allergy Clin Immunol 1990; 85: 671-683

${ }^{2}$ Cypar D, Stark J, Lemanske Jr RF. The impact of respiratory infections on asthma. Ped Clin North Am 1992; 39: 1259-1276

${ }^{3}$ Johnston SL, Pattemore PK, Sanderson G, Smith S, Lampe F, Josephs LK, Symington P, O'Toole S, Myint SH, Tyrrell DAJ, Holgate ST. Community study of the role of virus infections in exacerbations of asthma in 9-11 year old children. Br Med J 1995; 310: $1225-1228$

${ }^{4}$ Johnston SL, Pattemore PK, Sanderson G, Smith S, Campbell MJ, Josephs LK, Cunningham A, Robinson BS, Myint SH, Ward MJ, Tyrrell DAJ, Holgate ST. The association of upper respiratory infections with hospital admissions for asthma in adults and children: a time trend analysis. Am J Resp Crit Care Med 1996; 154: $654-660$

${ }^{5}$ Johnston SL. The role of viral and atypical bacterial pathogens in asthma pathogenesis. Pediatr Pulmonol 1999; Supp 18: $141-$ 143

${ }^{6}$ McIntosh K, Ellis EF, Hoffman LS, Lybass TG, Eller JJ, Fulginiti VA. The association of viral and bacterial respiratory infections with the exacerbation of wheezing in young asthmatic children. J Pediatr 1973; 82: $578-590$

${ }^{7}$ Pattemore PK, Johnson SL, Bardin PG. Viruses as precipitants of asthma symptoms. I. Epidemiology. Clin Exp Allergy 1992; 22: $325-336$

${ }^{8}$ Duff AL, Pomeranz ES, Gelber LE, Price W, Farris H, Hayden FG, Platts-Mills TAE, Heymann PW. Risk factors for acute wheezing in infants and children: viruses, passive smoke, and IgE antibodies to inhalant allergens. Pediatrics 1993; 92: 535-540

${ }^{9}$ Martinez FD, Morgan WJ, Wright AL, Holberg CJ, Taussig LM. Diminished lung function as a predisposing factor for wheezing respiratory illness in infants. N Engl J Med 1988; 319: 1112 - 1117

${ }^{10}$ Martinez FD, Morgan WJ, Wright AL, Holberg CJ, Taussig LM. Initial airway function is a risk factor for recurrent wheezing respiratory illness during the first 3 years of life. Am Rev Resp Dis 1991; 143: $312-316$

${ }^{11}$ Martinez FD, Wright AL, Taussig LM, Holberg CJ, Halonen M, Morgan WJ. Asthma and wheezing in the first six years of life. N Engl J Med 1995; 332: $133-137$

${ }^{12}$ Martinez FD, Stern DA, Wright AL, Taussig LM, Halonen M. Differential immune responses to acute lower respiratory illness in early life and subsequent development of persistent wheezing and asthma. J Allergy Clin Immunol 1998; 102: 915 - 920

${ }^{13}$ Gern JE, Galagan DM, Jarjour NN, Dick EC, Busse WW. Detection of rhinovirus RNA in lower airway cells during experimentally induced infection. Am J Respir Crit Care Med 1997; 155: 11591161

${ }^{14}$ Fraenkel DJ, Bardin PG, Sanderson G, Lampe F, Johnston SL, Holgate ST. Lower airways inflammation during rhinovirus colds in normal and asthmatic subjects. Am J Resp Crit Care Med 1995; 151: 879-886 
${ }^{15}$ Wohl MEB. Bronchiolitis. In: Chernick V, Kendrig Jr EL, eds. Disorders of the respiratory tract in children. 5th ed. W. B. Saunders, 1990: 360-370

${ }^{16}$ Neilson KA, Yunis EJ. Demonstration of respiratory syncytial virus in an autopsy series. Pediatr Pathol 1990; 10: 491-502

17 Wang SZ, Forsyth KD. Asthma and respiratory syncytial virus infection in infancy: is there a link? Clin Exp Allergy 1998; 28: 927-935

${ }^{18}$ Garofalo R, Kimpen JLL, Welliver RC, Ogra PL. Eosinophil degranulation in the respiratory tract during naturally acquired respiratory syncytial virus infection. J Pediatr 1992; 120: 28-32

${ }^{19}$ Garofalo R, Dorris A, Ahlstedt S, Welliver RC. Peripheral blood eosinophil counts and eosinophil cationic protein content of respiratory secretions in bronchiolitis: relationship to severity of disease. Pediatr Allergy Immunol 1994; 5: 111 - 117

${ }^{20}$ Kimpen JLL, Garofalo R, Welliver RC, Ogra PL. Activation of human eosinophils in vitro by respiratory syncytial virus. Pediatr Res 1992; 32: 160-164

${ }^{21}$ Noah TL, Becker S. Respiratory syncytial virus-induced cytokine production by a human bronchial epithelial cell line. Am J Physiol 1993; 265: L472 - L478

${ }^{22}$ Arnold R, Humbert B, Werchau H, Gallati H, Konig W. Interleukin-8, interleukin-6, and soluble tumour necrosis factor receptor type I release from a human pulmonary epithelial cell line (A549) exposed to respiratory syncytial virus. Immunology 1994; 82: $126-133$

${ }^{23}$ Becker S, Quay J, Soukup J. Cytokine (tumor necrosis factor, IL-6 and IL-8) production by respiratory syncytial virus-infected human alveolar macrophages. J Immunol 1991; 147: 4307-4312

${ }^{24}$ Levandowski R, Ou D, Jackson G. Acute-phase decrease of Tlymphocyte subsets in rhinovirus infection. J Infect Dis 1986; 153: $743-748$

${ }^{25}$ Levandowski R, Weaver C, Jackson G. Nasal secretion leucocyte populations determined by flow cytometry during acute rhinovirus infection. J Med Virol 1988; 25: $423-432$

${ }^{26}$ Folkerts G, Busse WW, Nijkamp FP, Sorkness R, Gern JE. Virusinduced airway hyperresponsiveness and asthma. Am J Respir Crit Care Med 1998; 157: 1708-1720

${ }^{27}$ Schwarze J, Hamelmann E, Bradley KL, Takeda K, Gelfand EW. Respiratory syncytial virus infection results in airway hyperresponsiveness and enhanced airway sensitization to allergen. J Clin Invest 1997; 100: 226-233

${ }^{28}$ Schwarze J, Cieslewicz G, Joetham A, Ikemura T, Hamelmann E, Gelfand EW. CD8 T cells are essential in the development of respiratory syncytial virus-induced lung eosinophilia and airway hyperresponsiveness. J Immunol 1999; 162: 4207-4211

${ }^{29}$ Coyle AJ, Erard F, Bertrand C, Walti S, Pircher H, Le Gros G. Virusspecific CD8+ cells can switch to interleukin 5 production and induce airway eosinophilia. J Exp Med 1995; 181: 1229-1233

${ }^{30}$ Schwarze J, Cieslewicz G, Hamelmann E, Joetham A, Schultz LD, Lamers MC, Gelfand EW. IL-5 and eosinophils are essential for the development of airway hyperresponsiveness following acute respiratory syncytial virus infection. J Immunol 1999; 162: 2997-3004

${ }^{31}$ Fryer AD, Jacoby DB. Parainfluenza virus infection damages inhibitory M2 muscarinic receptors on pulmonary parasympathetic nerves in the guinea-pig. Br J Pharmacol 1991; 102: $267-271$

${ }^{32}$ Fryer AD, Jacoby DB. Muscarine receptors and control of airway smooth muscle. Am J Resp Crit Care Med 1998; 158: S154-S160

${ }^{33}$ Fryer AD, el-Fakahany EE, Jacoby DB. Parainfluenza virus type 1 reduces the affinity of agonists for muscarinic receptors in guinea-pig lung and heart. Eur J Pharmacol 1990; 181: 51 - 58

${ }^{34}$ Folkerts G, van der Linde HJ, Nijkamp FP. Virus-induced airway hyperresponsiveness in guinea pigs is related to a deficiency in nitric oxide. J Clin Invest 1995; 95: 26-30
${ }^{35}$ Colasurdo GN, Hemming VG, Prince GA, Loader JE, Graves JP, Larsen GL. Human respiratory syncytial virus affects nonadrenergic noncholinergic inhibition in cottoc rat airways. Am J Physiol 1995; 268: L1006-1011

${ }^{36}$ Borson DB, Brokaw JJ, Sekizawa K, McDonald DM, Nadel JA. Neutral endopeptidase and neurogenic inflammation in rats with respiratory infections. J Appl Physiol 1989; 66: 2653-2658

${ }^{37}$ Dusser DJ, Jacoby DB, Djokic TD, Rubinstein I, Borson DB, Nadel JA. Virus induces airway hyperresponsiveness to tachykinins: role of neutral endopeptidase. J Appl Physiol 1989; 67: 15041511

38 Jacoby DB, Tamaoki J, Borson DB, Nadel JA. Influenza infection causes airway hyperresponsiveness by decreasing enkephalinase. J Appl Physiol 1988; 64: 2653 - 2658

${ }^{39}$ Maggi CA, Patacchini R, Perretti F, Meini S, Manzini S, Santicioli P, Del Bianco E, Meli A. The effect of thiorphan and epithelium removal on contractions and tachykinin release produced by activation of capsaicin-sensitive afferents in the guinea-pig isolated bronchus. Naunyn Schmiedebergs Arch Pharmacol 1990; 341: $74-79$

${ }^{40}$ Yang XX, Powell WS, Hojo M, Martin JG. Hyperpnea-induced bronchoconstriction is dependent on tachykinin-induced cysteinyl leukotriene synthesis. J Appl Physiol 1997; 82: 538-544

${ }^{41}$ Couture R, Cuello AC. Studies on the trigeminal antidromic vasodilatation and plasma extravasation in the rat. J Physiol 1984; 346: $273-285$

${ }^{42}$ Coles SJ, Neill KH, Reid LM. Potent stimulation of glycoprotein secretion in canine trachea by substance P. J Appl Physiol 1984; 57: $1323-1327$

43 Piedimonte G, Hoffman JI, Husseini WK, Snider RM, Desai MC, Nadel JA. NK1 receptors mediate neurogenic inflammatory increase in blood flow in rat airways. J Appl Physiol 1993; 74: $2462-2468$

${ }^{44}$ Grünberg K, Kuijpers EA, de Klerk EP, de Gouw HW, Kroes AC, Dick EC, Sterk PJ. Effects of experimental rhinovirus 16 infection on airway hyperresponsiveness to bradykinin in asthmatic subjects in vivo. Am J Respir Crit Care Med 1997; 155: 833-838

45 Joos GF, Pauwels RA. The in vivo effect of tachykinins on airway mast cells of the rat. Am Rev Respir Dis 1993; 148: 922 - 926

${ }^{46}$ Shaheen S, Aaby P, Hall A, Barker D, Heyes C, Shiell A, Goudiaby A. Measles and atopy in Guinea-Bissau. Lancet 1996; 347: $1792-$ 1796

47 Shirakawa T, Enomoto T, Shimazu SI, Hopkin J. The inverse association between tuberculin response and atopic disorder. Science 1997; 275: 77-79

${ }^{48}$ von Mutius E, FD M, Frirzsch C, Nicolai T, Reitmann P, Thiemann H. Skin test reactivity and number of siblings. Br Med J 1994; 308: $692-695$

49 Strachan D. Hay fever, hygiene, and household size. Br Med J 1989; 299: 1259-1260

${ }^{50}$ Braun-Fahrländer C, Gassner M, Grize L, Neu U, Sennhauser F, Varonier H, Vuille J, Wüthrich B. Prevalence of hay fever and allergic sensitization in farmer's children and their peers living in the same rural community. Clin Exp Allergy 1999; 29: 28 - 34

${ }^{51}$ Erb K, Holloway J, Sobeck A, Moll H, Le Gros G. Infection of mice with Mykobacterium bovis-bacillus Calmette-Guerin (BCG) suppresses allergen-induced airway eosinophilia. J Exp Med 1998; 187: $561-569$

${ }^{52}$ Herz U, Gerhold K, Grüber C, Braun A, Wahn U, Renz H, Paul K. BCG infection suppresses allergic sensitization and development of increased airway reactivity in an animal model. J Allergy Clin Immunol 1998; 102: 867-874

${ }^{53}$ Michele T, Kuperman D, Bishai W, Wills-Karp M. BCG infection reduces antigen-induced airway hyperresponsiveness in genetically susceptible mice. Am J Resp Crit Care Med 1998; 157: A244 
${ }^{54}$ Kraft M, Cassell G, Henson J, Watson H, Williamson J, Marmion B, Gaydos C, Martin R. Detection of mycoplasma pneumoniae in the airways of adults with chronic asthma. Am J Resp Crit Care Med 1998; 158: 998 - 1001

${ }^{55}$ Kraft M, Hamid Q, Cassell G, Gaydos C, Metze T, Duffy L, Rex M, Pak J, Martin R. Mycoplasma and chlamydia cause increased airway inflammation that is responsive to clarithromycin. Am J Resp Crit Care Med 1999; 159: A517

${ }^{56}$ Frick OL, German DF, Mills J. Development of allergy in children. I. Association with virus infections. J Allergy Clin Immunol 1979; 63: $228-241$

57 Sigurs N, Bjarnason R, Sigurbergsson F, Kjellman B, Björkstén B. Asthma and immunoglobulin $\mathrm{E}$ antibodies after respiratory syncytial virus bronchiolitis: a prospective cohort study with matched controls. Pediatrics 1995; 95: 500-505

${ }^{58}$ Sims DG, Downham MA, Gardner PS, Webb JK, Weightman D. Study of 8-year-old children with a history of respiratory syncytial virus bronchiolitis in infancy. Br Med J 1978; 1: 11 - 14

${ }^{59}$ Pullan CR, Hey EN. Wheezing, asthma and pulmonary dysfunction 10 years after infection with respiratory syncytial virus in infancy. Br Med J 1982; 284: 1665 - 1669

${ }^{60}$ Hall CB, Hall WJ, Gala CL, MaGill FB, Leddy JP. Long-term prospective study in children after respiratory syncytial virus infection. J Pediatr 1984; 105: 358 - 364

${ }^{61}$ Stein RT, Sherrill D, Morgan WJ, Holberg CJ, Halonen M, Taussig LM, Wright AL, Martinez FD. Respiratory syncytial virus in early life and risk of wheeze and allergy by age 13 years. Lancet 1999; 354: $541-545$

62 Stein RT, Holberg CJ, Morgan WJ, Wright AL, Lombardi E, Taussig LM, Martinez FD. Peak flow variability, methacholine responsiveness and atopy as markers for detecting different wheezing phenotypes in childhood. Thorax 1997; 52: 946-952

63 Riedel F, Krause A, Slenczka W, Rieger CHL. Parainfluenza-3virus infection enhances allergic sensitization in the guinea pig. Clin Exper Allergy 1996; 26: 603 - 609

${ }^{64}$ Leibovitz E, Freihorst J, Piedra PA, Ogra PL. Responses to inhaled ragweed antigen in experimentally induced infection with respiratory syncytial virus. Implication in virally induced allergy. Int Arch Allergy Appl Immunol 1988; 86: 112-116

${ }^{65}$ Freihorst J, Piedra PA, Okamoto J, Ogra PL. Effect of respiratory syncytial virus infection on the uptake of and immune response to other inhaled antigens. Proc Soc Exp Biol Med 1988; 188: $191-197$

${ }^{66}$ Schwarze J, Cieslewicz G, Hamelmann E, Joetham A, Schultz LD, Lamers MC, Gelfand EW. IL-4 and IL-5 during respiratory syncytial virus infection are critical for airway hyperresponsiveness following airway sensitization. Am J Resp Crit Care Med 2000; in print

${ }^{67}$ Schwarze J, Makela M, Cieslewicz G, Dakhama A, Lahn M, Ikemura T, Joetham A, Gelfand EW. Transfer of the enhancing effect of respiratory syncytial virus infection on subsequent allergic airway sensitization by T lymphocytes. J Immunol 1999; 163: $5729-5734$

68 Alwan WH, Kozlowska WJ, Openshaw PJM. Distinct types of lung disease caused by functional subsets of antivirual T cells. J Exp Med 1994; 179: 81 - 89

${ }^{69}$ Schwarze J, Hamelmann E, Cieslewicz G, Tomkinson A, Joetham A, Bradley K, Gelfand EW. Local treatment with IL-12 is an effective inhibitor of airway hyperresponsiveness and lung eosinophilia after airway challenge in sensitized mice. J Allergy Clin Immunol 1998; 102: 86-93

${ }^{70}$ Broide D, Schwarze J, Tighe H, Gifford T, Nguyen M, Malek S, Van Uden J, Martin-Orozco E, Gelfand E, RAZ ER. Immunostimulatory DNA sequences inhibit IL-5, eosinophilic inflammation, and airway hyperresponsiveness in mice. J Immunol 1998; 161: $7054-7062$
Dr. med. J. Schwarze

Klinik für Kinder- und Jugendmedizin im St. Josef-Hospital Bochum

Universitätsklinik

Alexandrinenstr. 5

44791 Bochum

E-mail: juergen.schwarze@ruhr-uni-bochum.de 